\title{
Low-dose cadmium activates the JNK signaling pathway in human renal podocytes
}

\author{
XIAOCUI CHEN ${ }^{1}$, YINGHUA XU ${ }^{2}$, ZUOWANG CHENG $^{2}$, HONG SU $^{3}$, XIU LIU $^{4}$, \\ DONGMEI XU ${ }^{3}$, CAROLYN KAPRON ${ }^{5}$ and JU LIU ${ }^{1}$
}

\author{
${ }^{1}$ Medical Research Center, Shandong Provincial Qianfoshan Hospital, Shandong University, Jinan, Shandong 250014; \\ ${ }^{2}$ School of Graduate Studies, Taishan Medical College, Tai'an, Shandong $271000 ;{ }^{3}$ Department of Nephrology, \\ Shandong Provincial Qianfoshan Hospital, Shandong University, Jinan, Shandong 250014; ${ }^{4}$ Department of Cardiography, \\ Binzhou Medical University Affiliated Hospital, Binzhou, Shandong 256600, P.R. China; \\ ${ }^{5}$ Department of Biology, Trent University, Peterborough, ON K9J 7B8, Canada
}

Received January 14, 2017; Accepted January 24, 2018

DOI: $10.3892 / \mathrm{ijmm} .2018 .3445$

\begin{abstract}
Cadmium $(\mathrm{Cd})$ is an environmental toxin. Our previous study demonstrated that low-dose $\mathrm{Cd}$ damages the integrity of the glomerular filtration barrier (GFB); however, the underlying mechanisms are poorly understood. Podocytes are a major component of the GFB, which regulate the passage of proteins. The present study aimed to investigate the effects of low-dose Cd on human renal podocytes (HRPs). HRPs were treated with $\mathrm{Cd}$ and activation of the c-Jun N-terminal kinase (JNK) pathway was examined by western blot analysis. Proliferation, viability and apoptosis of HRPs were evaluated by MTT assay, trypan blue exclusion assay and flow cytometry, respectively. The properties of HRPs were validated by immunofluorescence staining and Phalloidin-labeling. The results indicated that $4 \mu \mathrm{M} \mathrm{Cd}$ may activate the JNK pathway, and increase the protein expression levels of c-Jun and c-Fos. However, proliferation, viability, apoptosis and alignment of the F-actin cytoskeleton in HRPs were not significantly affected by Cd treatment, with or without SP600125 pretreatment. In addition, the expression levels of CD2-associated protein and synaptopodin, which are differentiation markers of HRPs, remained unchanged following $\mathrm{Cd}$ treatment. These results indicated that low-dose $\mathrm{Cd}$ activates the JNK pathway but does not significantly affect HRP function.
\end{abstract}

Correspondence to: Professor Dongmei Xu, Department of Nephrology, Shandong Provincial Qianfoshan Hospital, Shandong University, 16766 Jingshi Road, Jinan, Shandong 250014, P.R. China E-mail: xudongmei63@163.com

Professor Ju Liu, Medical Research Center, Shandong Provincial Qianfoshan Hospital, Shandong University, 16766 Jingshi Road, Jinan, Shandong 250014, P.R. China

E-mail: ju.liu@sdu.edu.cn

Key words: cadmium, c-Jun N-terminal kinase signaling, podocytes, F-actin

\section{Introduction}

Cadmium (Cd) is an environmentally abundant toxic metal, which is considered a serious threat to human health (1). Cd has a long biological half-life, and exhibits organ accumulation. One of the primary target organs of Cd is the kidney (2). In addition to accumulation in proximal tubular cells, $\mathrm{Cd}$ exposure directly damages the glomerulus, thus resulting in proteinuria (3). However, little is currently known regarding the molecular mechanisms underlying its cytotoxic effects.

The glomerular filtration barrier (GFB) consists of fenestrated endothelial cells, the glomerular basement membrane and podocyte slit diaphragms (4). It achieves size selectivity of the glomerular filter, permitting filtration of water and small-sized solutes in the plasma (5). Surrounding the glomerular capillaries, podocytes are highly specialized epithelial cells that maintain the structural and functional integrity of the GFB. As interdigitating foot processes separated by a slit diaphragm, podocytes regulate the passage of proteins from the capillary lumen to Bowman's space (6). Podocyte dysfunction impairs size selectivity of the glomerular filter, thus leading to proteinuria, hypoalbuminuria and edema (5). In addition, podocyte phenotype is determined by the expression of CD2-associated protein (CD2AP) and synaptopodin (7). Podocyte dedifferentiation over the course of collapsing focal segmental glomerulosclerosis results in the loss of synaptopodin and cytoskeleton markers $(8,9)$. Oxidized low-density lipoprotein stimulation re-organizes F-actin filaments and promotes podocyte migration in a focal adhesion kinase (FAK)-dependent manner (10).

Mitogen-activated protein kinases (MAPKs) are a family of serine/threonine protein kinases, which regulate numerous biological cellular processes, including cell proliferation, apoptosis and F-actin cytoskeletal formation (11). The three major MAPK pathways in mammalian cells are extracellular signal-regulated kinases, c-Jun N-terminal kinase (JNK) and p38 MAPK (11). JNK, also termed stress-activated protein kinase (SAPK), is activated by stress signals, including heat shock, ultraviolet light, osmotic stress and metabolic toxins (12-14). Activated JNK translocates to the nucleus and activates c-Jun and c-Fos, thus resulting in the formation 
of activating protein-1 (AP-1) (15). AP-1 recognizes either 12-O-tetradecanoylphorbol-13-acetate response elements or cAMP response elements, and activates the expression of a series of stress-response genes (16).

The present study aimed to examine the effects of low-dose $\mathrm{Cd}(4 \mu \mathrm{M})$ on human renal podocytes (HRPs). The results indicated that $\mathrm{Cd}$ activates the JNK pathway, thus increasing c-Jun and c-Fos expression. However, Cd had no effect on proliferation, viability, apoptosis and alignment of the F-actin cytoskeleton of HRPs. In addition, treatment with SP600125, a JNK inhibitor, alongside Cd did not alter cell proliferation, viability, apoptosis and alignment of the F-actin cytoskeleton of HRPs compared with SP600125 treatment alone.

\section{Material and methods}

Cell culture. HRPs were originally provided by Dr MA Saleem (University of Bristol, Bristol, UK), and were cultured in RPMI-1640 medium (Corning, Inc., Corning, NY, USA) supplemented with $10 \%$ fetal bovine serum (Gibco ${ }^{\mathrm{TM}}$; Thermo Fisher Scientific, Inc., Waltham, MA, USA), $100 \mathrm{U} / \mathrm{ml}$ penicillin and $100 \mu \mathrm{g} / \mathrm{ml}$ streptomycin. The cells were grown in a humidified atmosphere containing $5 \% \mathrm{CO}_{2}$ at $37^{\circ} \mathrm{C} . \mathrm{CdCl}_{2}$ was purchased from Sigma-Aldrich; Merck KgaA (Darmstadt, Germany) and was dissolved in PBS, with a stock concentration of $1 \mathrm{mM}$. In the present study, $4 \mu \mathrm{M} \mathrm{CdCl}_{2}$ was considered low-dose treatment. SP600125 was purchased from Cell Signaling Technology, Inc. (Danver, MA, USA) and was dissolved in dimethyl sulfoxide. Cells were pretreated with $10 \mu \mathrm{M} \mathrm{SP} 600125$ for $1 \mathrm{~h}$ prior to treatment with Cd. Control cells were only treated with $10 \mu \mathrm{M}$ SP600125 for $1 \mathrm{~h}$. Experimental cells were pretreated with $10 \mu \mathrm{M} \mathrm{SP} 600125$ for $1 \mathrm{~h}$, and then were treated with $\mathrm{Cd}$ for 1 , 2, 6, 12 and $24 \mathrm{~h}$. All treatments were at $37^{\circ} \mathrm{C}$.

Western blotting. Protein samples were extracted from HRPs using radioimmunoprecipitation assay buffer $[20 \mathrm{mM}$ Tris (pH 7.5), $150 \mathrm{mM} \mathrm{NaCl}, 50 \mathrm{mM} \mathrm{NaF}, 1 \% \mathrm{NP}-40$, $0.1 \%$ deoxycholate, $0.1 \%$ sodium dodecyl sulfate, $1 \mathrm{mM}$ EDTA] supplemented with the protease inhibitors aprotonin $(1 \mu \mathrm{g} / \mathrm{ml})$, leupeptin $(10 \mu \mathrm{g} / \mathrm{ml})$ and phenylmethylsulfonyl fluoride $(1 \mathrm{mM})$. Equal amounts of protein [40 $\mu \mathrm{g}$; protein concentration was determined using BCA assay (Bio-Rad, Hercules, CA, USA)] were separated by $8 \%$ SDS-PAGE (Beyotime Institute of Biotechnology, Haimen, China) and were then transferred onto a polyvinylidene fluoride membrane. The membrane was blocked with 5\% non-fat milk in Tris-buffered saline containing 1\% Tween-20 (TBST; Cell Signaling Technology, Inc.) at room temperature for $2 \mathrm{~h}$ prior to incubation with primary antibodies overnight at $4^{\circ} \mathrm{C}$. After washing three times with TBST, the membrane was incubated with a peroxidase-conjugated secondary antibody at room temperature for $2 \mathrm{~h}$. The primary antibodies used in the present study were as follows: Rabbit anti-SAPK/JNK (9258), rabbit anti-phosphorylated (p)-SAPK/JNK (4668), rabbit anti-c-Fos (2250), rabbit anti-c-Jun (9165) and rabbit anti-GAPDH (2118) (Cell Signaling Technology, Inc.). The secondary antibody used was goat anti-rabbit immunoglobulin G (7074; Cell Signaling Technology, Inc.). The immunoblots were developed using enhanced chemiluminescence reagents (EMD Millipore, Billerica, MA, USA), and relative blot intensities were semi-quantified using ImageJ software (version 1.4.3.67; National Institutes of Health, Bethesda, MA, USA).

Cell proliferation and viability assay. HRP proliferation was evaluated using the MTT assay kit (Cayman Chemical Company, Ann Arbor, MI, USA) according to the manufacturer's protocol. Briefly, HRPs were plated at a density of $9 \times 10^{3}$ cells/well in a 96-well plate and were cultured overnight. Subsequently, the cells were treated with Cd, SP600125 or a combination of Cd and SP600125 for $24 \mathrm{~h}$, after which $10 \mu \mathrm{l}$ MTT solution was added to each well and incubated for $4 \mathrm{~h}$ at $37^{\circ} \mathrm{C}$. The combination of SP600125 and Cd treatment was as follows: cells were pretreated with SP600125 for $1 \mathrm{~h}$, and then co-treated with Cd and SP600125 for $24 \mathrm{~h}$. The crystals were solubilized by the addition of $110 \mu \mathrm{l}$ DMSO, and colorimetric intensity was analyzed using a 96-well plate reader (Molecular Devices, LLC, Sunnyvale, CA, USA) at a wavelength of $490 \mathrm{~nm}$. HRP viability was assessed using a trypan blue exclusion assay. Following treatment, HRPs were washed and incubated in $0.05 \%$ trypsin for $2 \mathrm{~min}$ at $37^{\circ} \mathrm{C}$. Subsequently, the cells were disaggregated into a single cell suspension and diluted 9:1 with 0.4\% trypan blue (Beijing Solarbio Science \& Technology Co., Ltd., Beijing, China). The percentage of unstained cells was determined under an OLYMPUS CKX41 microscope (Olympus Corporation, Tokyo, Japan).

Immunofluorescence. HRPs were grown until confluent (80\%) on fibronectin-coated glass chamber slides and were then treated with $4 \mu \mathrm{M} \mathrm{Cd}$ for $24 \mathrm{~h}$. The medium was then aspirated, and monolayers were washed with PBS, fixed with $4 \%$ paraformaldehyde at room temperature for $20 \mathrm{~min}$ and washed three times with PBS for $15 \mathrm{~min}$. Subsequently, the cells were permeabilized with $0.3 \%$ Triton X-100 for $10 \mathrm{~min}$, washed three times with PBS for $15 \mathrm{~min}$ and incubated with a rabbit polyclonal antibody against CD2AP (1:50; \#2135; Cell Signaling Technology, Inc.) or synaptopodin (1:50; ab224491; Abcam, Cambridge, MA, USA) overnight at $4^{\circ} \mathrm{C}$. The cells were then incubated with an Alexa Fluor 546-labeled donkey anti rabbit secondary antibody (1:200; A10040; Thermo Fisher Scientific, Inc.) for $2.5 \mathrm{~h}$ at room temperature. Images of the slides were captured using an Olympus FSX100 Imaging system (Olympus Corporation) with an excitation wavelength of $546 \mathrm{~nm}$.

Annexin V-fluorescein isothiocyanate (FITC)/propidium iodide (PI) analysis. Apoptosis of the HRPs was determined by Annexin V-FITC and PI staining using an assay kit (Neobioscience, Shenzhen, China) according to the manufacturer's protocol. Briefly, following treatment with $\mathrm{Cd}$, SP600125 or a combination of Cd and SP600125 for $24 \mathrm{~h}$, cells were trypsinized and resuspended into a single cell suspension. Subsequently, $1 \times 10^{6}$ cells were stained with Annexin V-FITC $(0.025 \%)$ for $3 \mathrm{~min}$ and PI $(20 \mu \mathrm{g} / \mathrm{ml})$ for $10 \mathrm{~min}$ at room temperature in the dark. Positive staining of the cells was detected using a FACSAria II flow cytometer, and data were analyzed using the FACSDiva acquisition and analysis software (v6.1.3) (both from BD Biosciences, San Jose, CA, USA).

Phalloidin-labeling. HRPs were grown until confluent $(80 \%)$ on fibronectin-coated glass chamber slides. Following exposure to $4 \mu \mathrm{M} \mathrm{Cd}$, SP600125 or a combination of $4 \mu \mathrm{M} \mathrm{Cd}$ and 
$\mathbf{A}$

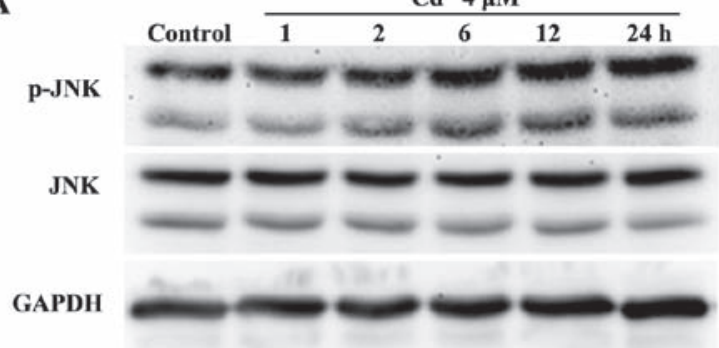

C

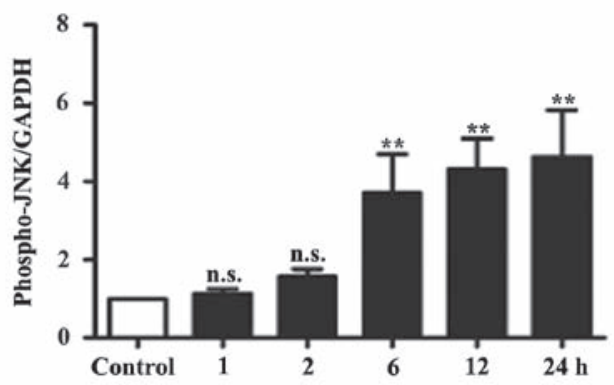

B

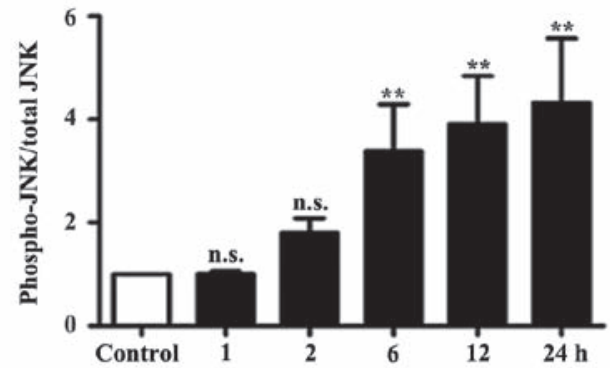

D

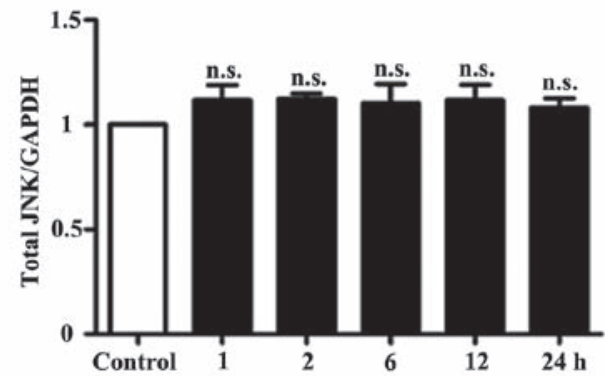

Figure 1. Cd activates JNK signaling in HRPs. (A) Representative blots of p-JNK and total JNK from HRPs treated with $4 \mu \mathrm{M}$ Cd at various time-points. GAPDH was used as a loading control. Densitometric analyses of (B) p-JNK/total JNK, (C) p-JNK/GAPDH, and (D) total JNK/GAPDH. n=3. *P<0.05 and ${ }^{* *} \mathrm{P}<0.01$ vs. the control group. Cd, cadmium; HRPs, human renal podocytes; JNK, c-Jun N-terminal kinase; n.s., not significant; p-JNK, phosphorylated-JNK.

SP600125 for $24 \mathrm{~h}$, the medium was aspirated and the monolayer was fixed for $5 \mathrm{~min}$ in $3.7 \%$ formaldehyde solution in PBS. The cells were then permeabilized with $0.3 \%$ Triton X-100 in PBS, and stained with $5 \mu \mathrm{g} / \mathrm{ml}$ phalloidin-tetramethylrhodamine B isothiocyanate (Sigma-Aldrich; Merck KGaA) in PBS for $1 \mathrm{~h}$ at room temperature. DAPI staining was used to visualize the nuclei. Images were captured using a confocal microscope (LSM 880; Carl Zeiss AG, Oberkochen, Germany).

Statistical analyses. Data are presented as means \pm SD. Statistical significance was assessed using one-way analysis of variance followed by Tukey's post hoc test or Student's t-test. Statistical analyses were performed using SPSS 17.0 statistical software package (SPSS Inc., Chicago, IL, USA). P $<0.05$ was considered to indicate a statistically significant difference.

\section{Results}

Low-dose Cd activates the JNK pathway in HRPs. Cd activates JNK signaling and stimulates downstream effectors of JNK in HepG2 cells (17). In the present study, the effects of $4 \mu \mathrm{M} \mathrm{Cd}$ exposure on the JNK pathway in HRPs were determined by western blotting. The results indicated that the expression levels of p-JNK were significantly increased following treatment of HRPs with $4 \mu \mathrm{M} \mathrm{Cd}$ for 6,12 and $24 \mathrm{~h}$. Conversely, the protein expression levels of total JNK and the internal control GAPDH remained unchanged (Fig. 1). In addition, the protein expression levels of c-Jun and c-Fos were significantly increased by $\mathrm{Cd}$ treatment (Fig. 2).

Low-dose Cd exposure does not affect proliferation and the expression of cell type-specific HRP markers. $\mathrm{Cd}$ inhibits the proliferation of numerous cell types $(18,19)$. In the present study, the MTT assay was performed to examine the effects of $\mathrm{Cd}$ on HRP proliferation. The results indicated that $4 \mu \mathrm{M} \mathrm{Cd}$ did not significantly inhibit HRP proliferation after $24 \mathrm{~h}$ (Fig. 3A). The results of the trypan blue exclusion assay also demonstrated that HRP viability remained unchanged following $\mathrm{Cd}$ treatment (Fig. 3B). Specific markers of podocytes include CD2AP and synaptopodin (7). In response to extracellular stimuli, podocytes may undergo a phenotypic alteration, thus resulting in the loss of terminal differentiation markers $(20,21)$. HRP characteristics were examined by immunofluorescence staining for CD2AP and synaptopodin. CD2AP and synaptopodin expression was unchanged in HRPs following $24 \mathrm{~h}$ exposure to $4 \mu \mathrm{M}$ $\mathrm{Cd}$ (Fig. 3C). These results suggested that low-dose Cd exposure may not alter the proliferation, viability and phenotype of HRPs.

Effects of low-dose Cd combined with SP600125 on HRP proliferation and viability. SP600125 is a specific inhibitor of the JNK pathway (22). Co-treatment with $10 \mu \mathrm{M} \mathrm{SP600125} \mathrm{for}$ $1 \mathrm{~h}$ inhibited Cd-induced phosphorylation of JNK (Fig. 4A). Conversely, HRP proliferation and viability were similar in the group treated with a combination of SP600125 and Cd compared with in the group treated with SP600125 alone (Fig. 4B and C).

Effects of low-dose Cd exposure on HRP apoptosis. The JNK pathway mediates apoptotic responses in numerous cell types (23). Following exposure to $4 \mu \mathrm{M} \mathrm{Cd}$ for $24 \mathrm{~h}$, apoptosis of HRPs was examined by Annexin V-FITC/PI double-labeled flow cytometry. No significant alterations in the apoptotic rate were detected in Cd-treated HRPs (Fig. 5). In addition, the apoptotic rate of HRPs treated with a combination of SP600125 and Cd was similar to that of HRPs treated with SP600125 alone. These results indicated that $4 \mu \mathrm{M} \mathrm{Cd}$ does not affect apoptosis of HRPs.

Effects of low-dose Cd exposure on the F-actin cytoskeleton of HRPs. Reorganization of the cytoskeleton is a hallmark 
$\mathbf{A}$

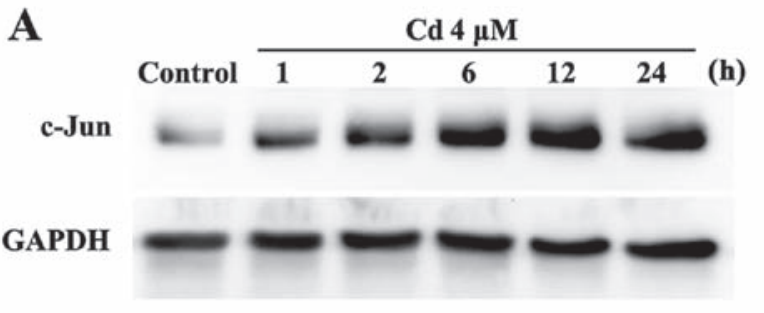

C

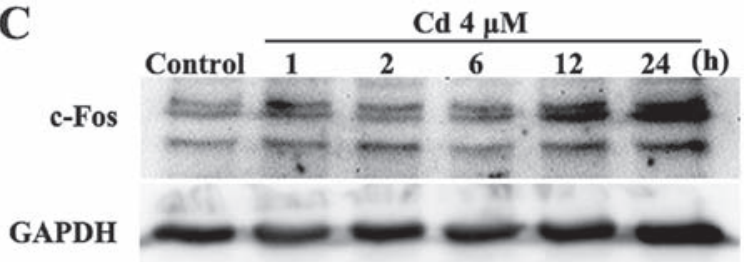

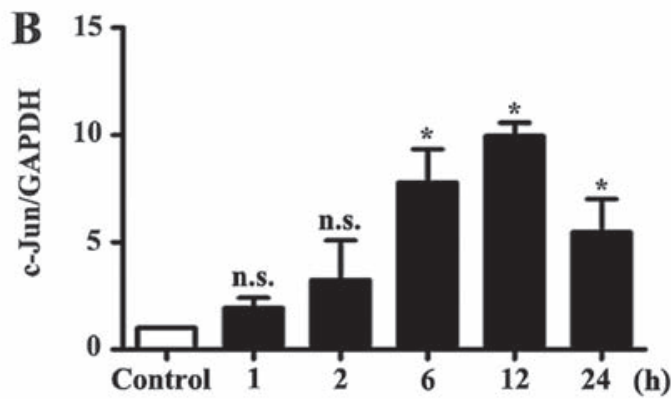

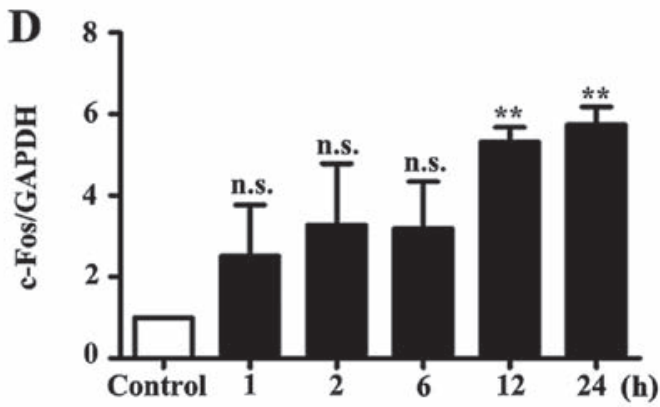

Figure 2. Cd increases the protein expression levels of c-Jun and c-Fos in HRPs. (A) Representative blots of c-Jun from HRPs treated with $4 \mu \mathrm{M}$ Cd at various time-points. GAPDH was used as a loading control. (B) Densitometric analysis of c-Jun/GAPDH. $n=3$. "P<0.05. (C) Representative blots of c-Fos from HRPs treated with $4 \mu \mathrm{M} \mathrm{Cd}$ at various time-points. GAPDH was used as a loading control. (D) Densitometric analysis of c-Fos/GAPDH. $\mathrm{n}=3$. *" $\mathrm{P}<0.01 \mathrm{vs}$. the control group. Cd, cadmium; HRPs, human renal podocytes; n.s., not significant.


C


Cd
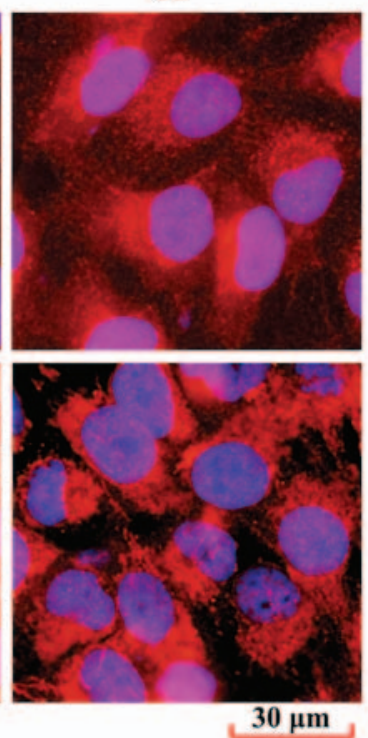

Figure 3. Effects of $\mathrm{Cd}$ on proliferation, viability and the expression of cell type-specific markers of HRPs. (A) MTT assay of HRPs treated with $4 \mu \mathrm{M}$ Cd for $24 \mathrm{~h}$. n=6. (B) Viability of HRPs following treatment with $4 \mu \mathrm{M} \mathrm{Cd}$ for 24 h. $n=3$. (C) Immunofluoresence staining of CD2AP and synaptopodin in HRPs treated with PBS (control) or $4 \mu \mathrm{M} \mathrm{Cd}$ for $24 \mathrm{~h}$. Magnification, $\mathrm{x} 200 . \mathrm{Cd}$, cadmium; CD2AP, CD2-associated protein; HRPs, human renal podocytes; n.s. non-significant.

$\mathbf{A}$

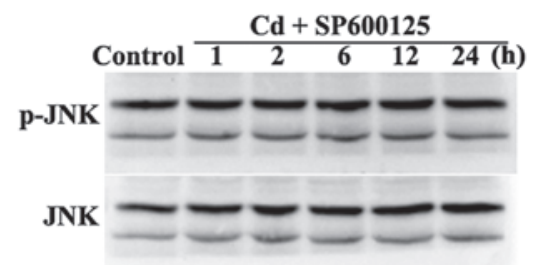

\section{GAPDH}

B

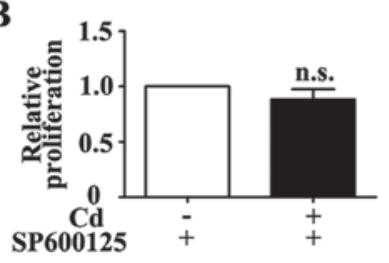

C

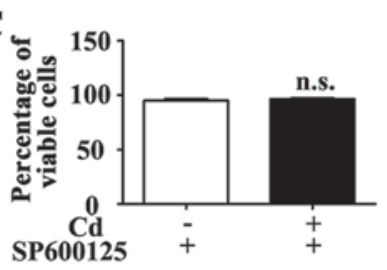

Figure 4. Effects of Cd in combination with SP600125 on HRP proliferation and viability. (A) Representative blots of p-JNK and total JNK from HRPs exposed to $4 \mu \mathrm{M} \mathrm{Cd}$ and pretreated with the JNK inhibitor SP600125. Cells treated with SP600125 alone were used as a control. (B) MTT assay of HRPs treated with $4 \mu \mathrm{M} \mathrm{Cd}$ for $24 \mathrm{~h}$ with or without SP600125 pretreatment. $\mathrm{n}=6$. (C) Cell viability of HRPs treated with $4 \mu \mathrm{M} \mathrm{Cd}$ for $24 \mathrm{~h}$ with or without SP600125 pretreatment. $\mathrm{n}=3$. Cd, cadmium; HRPs, human renal podocytes; JNK, c-Jun N-terminal kinase; n.s., not significant; p-JNK, phosphorylated-JNK.

of podocyte injury (24). The present study examined the podocyte cytoskeleton by immunostaining the F-actin cytoskeleton-interacting molecule phalloidin. The results demonstrated that F-actin arrangement in podocytes treated with $\mathrm{Cd}$ was not disrupted compared with in the control group (Fig. 6). In addition, SP600125, or a combined expo- 

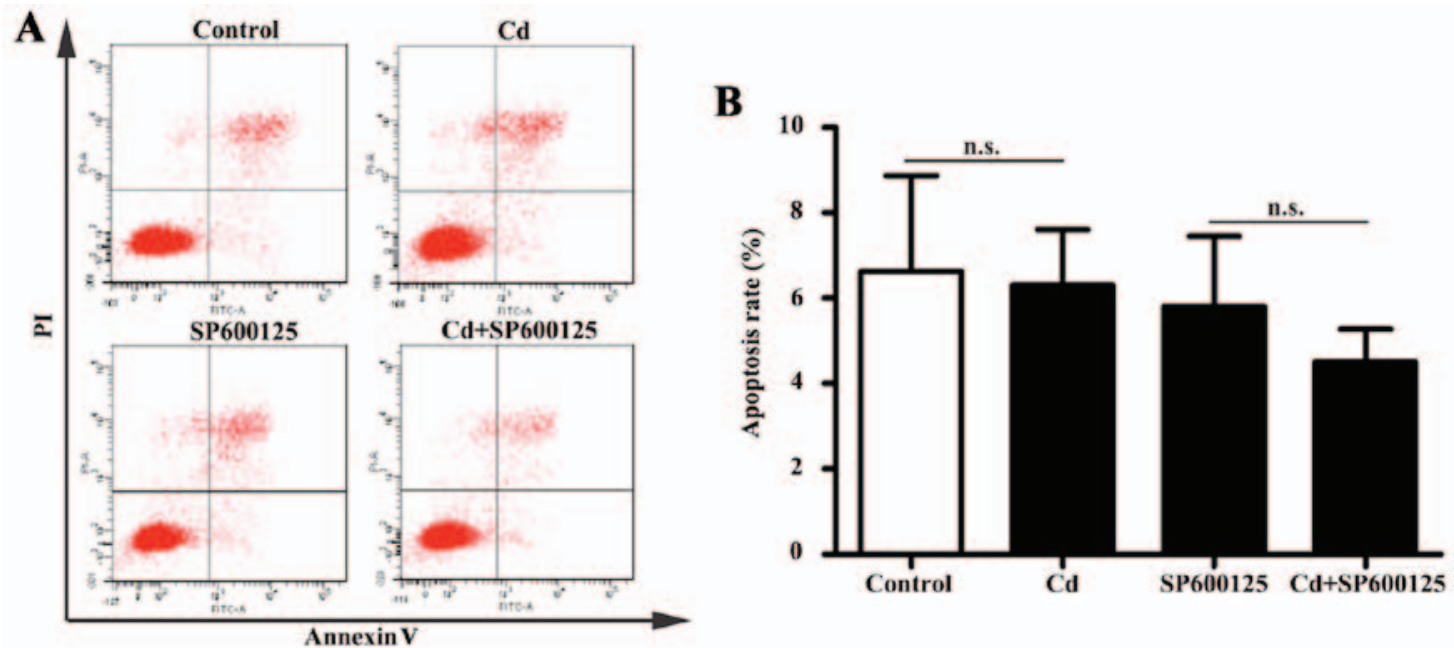

Figure 5. Effects of Cd on apoptosis of HRPs. (A) Representative image of flow cytometric detection of apoptotic HRPs. Cells were stained with Annexin V/PI following exposure to Cd, SP600125, or a combination of Cd and SP600125 for $24 \mathrm{~h}$. (B) Apoptotic rate of HRPs, as determined by flow cytometry. n=3. Cd, cadmium; HRPs, human renal podocytes; n.s., not significant; PI, propidium iodide.

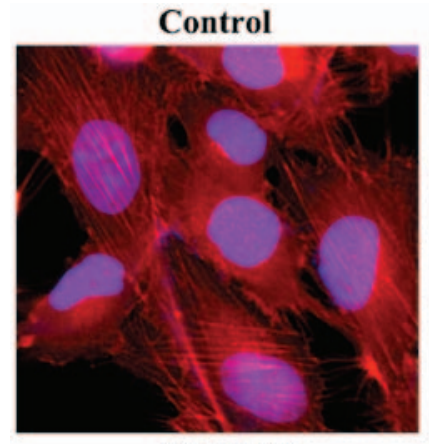

SP600125


Cd+SP600125

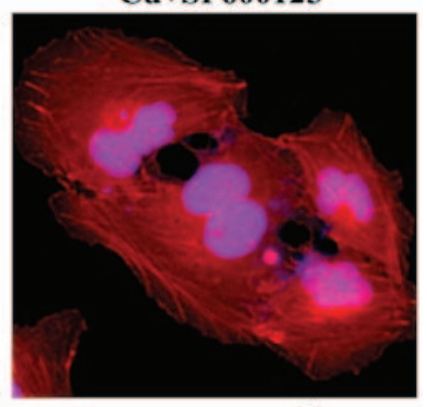

$30 \mu \mathrm{m}$

Figure 6. Effects of Cd on F-actin arrangement in HRPs. Immunofluorescent staining of F-actin with phalloidin-tetramethylrhodamine B isothiocyanate in HRPs treated with PBS (control), Cd, SP600125 or Cd + SP600125 for 24 h. Magnification, x200. Cd, cadmium; HRPs, human renal podocytes.

sure to SP600125 and Cd, did not significantly alter F-actin arrangement in podocytes.

\section{Discussion}

Cd has been reported to induce toxicological effects in the human kidney (25). The present study aimed to explore the effects of low-dose Cd on HRPs, which are the major component of the GFB. The results demonstrated that treatment with $4 \mu \mathrm{M} \mathrm{Cd}$ increased phosphorylation of JNK, and the expression levels of c-Jun and c-Fos. However, Cd treatment did not affect the proliferation, viability, apoptosis and cytoskeleton of HRPs. In addition, inhibition of the JNK pathway did not significantly affect Cd-treated HRPs. These findings indicated that $\mathrm{Cd}$ activates the JNK pathway in podocytes but does not induce cytotoxic effects.

Depending on the experimental settings, $\mathrm{Cd}$ may differentially affect specific cell types in the glomerulus $(12,26)$. As previously reported, although the vascular system is the primary target of $\mathrm{Cd}$ toxicity, a low concentration of $\mathrm{Cd}$ does not affect the proliferation and apoptosis of human umbilical vein endothelial cells or human renal glomerular endothelial cells (HRGECs) (26-28). However, Cd may increase apoptosis of human renal mesangial cells (HRMCs) and decrease their proliferation (12). Podocytes are a terminally differentiated and highly specialized cell type in the glomerulus. Previous studies have suggested that podocyte proliferation may not be affected in response to injury $(29,30)$. In addition, $\mathrm{Cd}$ affects cellular functions other than proliferation and apoptosis. A previous study demonstrated that low-dose $\mathrm{Cd}$ induces vascular hyperpermeability and disruption of endothelial barrier integrity via the Cd-induced membrane dissociation of vascular endothelial cadherin and $\beta$-catenin in HRGECs (26). Therefore, the effects of $\mathrm{Cd}$ on podocyte permeability require further investigation.

MAPKs are activated by numerous cellular stressors (11). Oxidative stress is one of the major mechanisms underlying $\mathrm{Cd}$ toxicity. The production of reactive oxygen species (ROS) induced by $\mathrm{Cd}$ causes severe toxic effects in numerous types of tissues and organs (31). JNK is one of the proteins activated in response to elevated ROS levels, which serves a critical role in the apoptotic process (32). At a concentration between 5 and $40 \mu \mathrm{M}, \mathrm{Cd}$ may induce apoptosis of BJAB cells by increasing DNA fragmentation and caspase-3 activity (33). In addition, at a concentration of $4 \mu \mathrm{M}, \mathrm{Cd}$ inhibits the proliferation of HRMCs via activation the JNK pathway (12). In the present study, treatment with $4 \mu \mathrm{M} \mathrm{Cd}$ increased phosphorylation of JNK, and c-Jun and c-Fos expression. However, activation of the JNK pathway did not induce apoptosis of HRPs. A previous study also indicated that $\mathrm{Cd}$ treatment increased p-JNK expression, but did not affect apoptosis of endothelial cells (26). Therefore, JNK-regulated cell functions may vary depending on cell type, stimulus, and the duration and strength 
of kinase activities $(31,34)$. In addition to MAPKs, the phosphatidylinositol 3-kinase/Akt pathway, hypoxia inducible factor-1 $\alpha$ and nuclear factor- $\kappa \mathrm{B}$ are involved in Cd-induced signal transduction (35-37). Activation of other signaling pathways may compensate the effects mediated by the JNK pathway.

An intact actin cytoskeleton is crucial for the maintenance of podocyte membrane tension, shape and glomerular function, and is associated with signaling events at the slit diaphragm (38). F-actin is one of the major components of the cytoskeleton (39). Synaptopodin is a proline-rich protein that is expressed in differentiated podocytes and orchestrates actin organization through its interaction with F-actin $(40,41)$. JNK is associated with cytoskeletal integrity, and is involved in 4-hydroxy-2-nonenal-mediated actin remodeling in endothelial cells (42). In the present study, Cd activated the JNK pathway, and increased the expression levels of c-Jun and c-Fos, but had no significant effect on synaptopodin distribution. A previous study also reported that the F-actin cytoskeleton was unchanged in HRMCs by $\mathrm{Cd}$ treatment, despite activation of the JNK pathway (12). Numerous signaling cascades may be involved in reorganization of the actin cytoskeleton (43). For example, high glucose-induced F-actin rearrangement in mouse podocytes was mediated by activation of the Fyn/Rho-associated coiled-coil forming protein kinase signaling pathway (44). Furthermore, the FAK/p38 signaling pathway regulates cytoskeletal arrangement in mouse podocytes (10). Therefore, activation of the JNK pathway may not be sufficient for rearrangement of the F-actin cytoskeleton in podocytes.

In conclusion, although low-dose $\mathrm{Cd}$ increased the phosphorylation of JNK, and the expression levels of c-Jun and c-Fos, it had no significant effect on proliferation, apoptosis and F-actin cytoskeletal rearrangement of HRPs. Further studies are required to address the molecular mechanisms underlying Cd-induced loss of glomerular barrier integrity.

\section{Acknowledgements}

Not applicable.

\section{Funding}

The present study was supported by grants from the Science and Technology Development Plan of Shandong Province (grant no. 2013GSF11805), the National Natural Science Foundation of China (grant no. 81370269) and the Shandong Taishan Scholarship (JL).

\section{Availability of data and materials}

The datasets used and/or analyzed during the current study are available from the corresponding author on reasonable request.

\section{Authors' contributions}

JL conceived and designed the experiments; XC, YX, ZC, HS, XL performed the experiments; JL, XC, DX and CMK analyzed the data; JL, XC and CMK wrote the paper.

\section{Ethics approval and consent to participate}

Not applicable.

\section{Consent for publication}

Not applicable.

\section{Competing interests}

The authors declare that they have no competing interests.

\section{References}

1. Eichler TE, Ransom RF and Smoyer WE: Differential induction of podocyte heat shock proteins by prolonged single and combination toxic metal exposure. Toxicol Sci 84: 120-128, 2005.

2. Liu J, Liu Y and Klaassen CD: Nephrotoxicity of $\mathrm{CdCl}_{2}$ and Cd-metallothionein in cultured rat kidney proximal tubules and LLC-PK1 cells. Toxicol Appl Pharmacol 128: 264-270, 1994.

3. Prozialeck WC, Edwards JR and Woods JM: The vascular endothelium as a target of cadmium toxicity. Life Sci 79: 1493-1506, 2006.

4. Pavenstädt H, Kriz W and Kretzler M: Cell biology of the glomerular podocyte. Physiol Rev 83: 253-307, 2003.

5. Sugano Y, Lindenmeyer MT, Auberger I, Ziegler U, Segerer S, Cohen CD, Neuhauss SC and Loffing J: The Rho-GTPase binding protein IQGAP2 is required for the glomerular filtration barrier. Kidney Int 88: 1047-1056, 2015.

6. Inoue $\mathrm{K}$ and Ishibe S: Podocyte endocytosis in the regulation of the glomerular filtration barrier. Am J Physiol Renal Physiol 309: F398-F405, 2015.

7. Testagrossa L, Azevedo Neto R, Resende A, Woronik V and Malheiros D: Immunohistochemical expression of podocyte markers in the variants of focal segmental glomerulosclerosis. Nephrol Dial Transplant 28: 91-98, 2013.

8. Barisoni L, Schnaper HW and Kopp JB: Advances in the biology and genetics of the podocytopathies: Implications for diagnosis and therapy. Arch Pathol Lab Med 133: 201-216, 2009.

9. Shankland SJ, Eitner F, Hudkins KL, Goodpaster T, D'Agati V and Alpers CE: Differential expression of cyclin-dependent kinase inhibitors in human glomerular disease: Role in podocyte proliferation and maturation. Kidney Int 58: 674-683, 2000.

10. Hu M, Fan M, Zhen J, Lin J, Wang Q, Lv Z and Wang R: FAK contributes to proteinuria in hypercholesterolaemic rats and modulates podocyte F-actin re-organization via activating p38 in response to ox-LDL. J Cell Mol Med 21: 552-567, 2017.

11. Seger R and Krebs EG: The MAPK signaling cascade. FASEB J 9: 726-735, 1995.

12. Chen X, Li J, Cheng Z, Xu Y, Wang X, Li X, Xu D, Kapron CM and Liu J: Low dose cadmium inhibits proliferation of human renal mesangial cells via activation of the JNK pathway. Int J Environ Res Public Health: Oct 7, 2016 (Epub ahead of print). doi: 10.3390/ijerph13100990.

13. Subramanian D, Bunjobpol W and Sabapathy K: Interplay between TAp73 protein and selected activator protein-1 (AP-1) family members promotes AP-1 target gene activation and cellular growth. J Biol Chem 290: 18636-18649, 2015.

14. Hess J, Angel P and Schorpp-Kistner M: AP-1 subunits: Quarrel and harmony among siblings. J Cell Sci 117: 5965-5973, 2004.

15. Davis RJ: Signal transduction by the JNK group of MAP kinases. Cell 103: 239-252, 2000.

16. Woodgett JR, Avruch J and Kyriakis JM: Regulation of nuclear transcription factors by stress signals. Clin Exp Pharmacol Physiol 22: 281-283, 1995.

17. Tsai JS, Chao CH and Lin LY: Cadmium activates multiple signaling pathways that coordinately stimulate Akt activity to enhance c-Myc mRNA stability. PLoS One 11: e0147011, 2016.

18. Liu C, Zhang R, Sun C, Zhang H, Xu C, Liu W, Gao W, Huang S and Chen L: Resveratrol prevents cadmium activation of Erk1/2 and JNK pathways from neuronal cell death via protein phosphatases $2 \mathrm{~A}$ and 5. J Neurochem 135: 466-478, 2015.

19. Gao X, Yu L, Moore AB, Kissling GE, Waalkes MP and Dixon D: Cadmium and proliferation in human uterine leiomyoma cells: Evidence of a role for EGFR/MAPK pathways but not classical estrogen receptor pathways. Environ Health Perspect 123: 331-336, 2015.

20. Barisoni L: Podocyte biology in segmental sclerosis and progressive glomerular injury. Adv Chronic Kidney Dis 19: 76-83, 2012.

21. Meyrier A: Mechanisms of disease: Focal segmental glomerulosclerosis. Nat Clin Pract Nephrol 1: 44-54, 2005. 
22. Bennett BL, Sasaki DT, Murray BW, O'Leary EC, Sakata ST, Xu W, Leisten JC, Motiwala A, Pierce S, Satoh Y, et al: SP600125, an anthrapyrazolone inhibitor of Jun N-terminal kinase. Proc Natl Acad Sci USA 98: 13681-13686, 2001.

23. Eichler T, Ma Q, Kelly C, Mishra J, Parikh S, Ransom RF Devarajan P and Smoyer WE: Single and combination toxic metal exposures induce apoptosis in cultured murine podocytes exclusively via the extrinsic caspase 8 pathway. Toxicol Sci 90: 392-399, 2006.

24. Shen X, Jiang H, Ying M, Xie Z, Li X, Wang H, Zhao J, Lin C, Wang Y, Feng S, et al: Calcineurin inhibitors cyclosporin A and tacrolimus protect against podocy te injury induced by puromycin aminonucleoside in rodent models. Sci Rep 6: 32087, 2016.

25. Provias JP, Ackerley CA, Smith C and Becker LE: Cadmium encephalopathy: A report with elemental analysis and pathological findings. Acta Neuropathol 88: 583-586, 1994.

26. Li L, Dong F, Xu D, Du L, Yan S, Hu H, Lobe CG, Yi F, Kapron CM and Liu J: Short-term, low-dose cadmium exposure induces hyperpermeability in human renal glomerular endothelial cells. J Appl Toxicol 36: 257-265, 2016.

27. Dong F, Guo F, Li L, Guo L, Hou Y, Hao E, Yan S, Allen TD and Liu J: Cadmium induces vascular permeability via activation of the p38 MAPK pathway. Biochem Biophys Res Commun 450: 447-452, 2014

28. Prozialeck WC, Edwards JR, Nebert DW, Woods JM, Barchowsky A and Atchison WD: The vascular system as a target of metal toxicity. Toxicol Sci 102: 207-218, 2008

29. Kriz W: Progressive renal failure--inability of podocytes to replicate and the consequences for development of glomerulosclerosis. Nephrol Dial Transplant 11: 1738-1742, 1996.

30. Rennke HG: How does glomerular epithelial cell injury contribute to progressive glomerular damage? Kidney Int Suppl 45: S58-S63, 1994.

31. Nemmiche S: Oxidative signaling response to cadmium exposure. Toxicol Sci 156: 4-10, 2017

32. Pineda-Molina E, Klatt P, Vázquez J, Marina A, García de Lacoba M, Pérez-Sala D and Lamas S: Glutathionylation of the p50 subunit of NF-kappaB: A mechanism for redox-induced inhibition of DNA binding. Biochemistry 40: 14134-14142, 2001

33. Nemmiche S, Chabane-Sari D, Kadri $M$ and Guiraud $P$ : Cadmium-induced apoptosis in the BJAB human B cell line: Involvement of PKC/ERK1/2/JNK signaling pathways in $\mathrm{HO}-1$ expression. Toxicology 300: 103-111, 2012.
34. Liu J and Kapron CM: Differential induction of MAP kinase signalling pathways by cadmium in primary cultures of mouse embryo limb bud cells. Reprod Toxicol 29: 286-291, 2010.

35. Lee JC, Son YO, Pratheeshkumar P and Shi X: Oxidative stress and metal carcinogenesis. Free Radic Biol Med 53: 742-757, 2012.

36. Zhang H, Li L, Wang Y, Dong F, Chen X, Liu F, Xu D, Yi F, Kapron CM and Liu J: NF- $\mathrm{BB}$ signaling maintains the survival of cadmium-exposed human renal glomerular endothelial cells. Int J Mol Med 38: 417-422, 2016.

37. Liu F, Wang B, Li L, Dong F, Chen X, Li Y, Dong X, Wada Y, Kapron CM and Liu J: Low-dose cadmium upregulates VEGF expression in lung adenocarcinoma cells. Int J Environ Res Public Health 12: 10508-10521, 2015.

38. Faul C, Asanuma K, Yanagida-Asanuma E, Kim K and Mundel P: Actin up: Regulation of podocyte structure and function by components of the actin cytoskeleton. Trends Cell Biol 17: 428-437, 2007.

39. Heinonen MT, Kanduri K, Lähdesmäki HJ, Lahesmaa R and Henttinen TA: Tubulin- and actin-associating GIMAP4 is required for IFN- $\gamma$ secretion during Th cell differentiation. Immunol Cell Biol 93: 158-166, 2015.

40. Müller-Krebs S, Kihm LP, Madhusudhan T, Isermann B, Reiser J, Zeier M and Schwenger V: Human RAGE antibody protects against AGE-mediated podocyte dysfunction. Nephrol Dial Transplant 27: 3129-3136, 2012.

41. Kim EY, Suh JM, Chiu YH and Dryer SE: Regulation of podocyte $\mathrm{BK}(\mathrm{Ca})$ channels by synaptopodin, Rho, and actin microfilaments. Am J Physiol Renal Physiol 299: F594-F604, 2010.

42. Usatyuk PV and Natarajan V: Role of mitogen-activated protein kinases in 4-hydroxy-2-nonenal-induced actin remodeling and barrier function in endothelial cells. J Biol Chem 279: 11789-11797, 2004

43. Ispanovic E and Haas TL: JNK and PI3K differentially regulate MMP-2 and MT1-MMP mRNA and protein in response to actin cytoskeleton reorganization in endothelial cells. Am J Physiol Cell Physiol 291: C579-C588, 2006.

44. Lv Z, Hu M, Ren X, Fan M, Zhen J, Chen L, Lin J, Ding N, Wang $Q$ and Wang R: Fyn mediates high glucose-induced actin cytoskeleton reorganization of podocytes via promoting ROCK activation in vitro. J Diabetes Res 2016: 5671803, 2016. 